\title{
Effects of Solar UV Radiation on Materials Used in Agricultural Industry in Salta, Argentina: Study and Characterization
}

\author{
Carlos Cadena1, Delicia Acosta ${ }^{2}$ \\ ${ }^{1}$ National Institute of Non-Conventional Energies, INENCO-CONICET, Universidad Nacional de Salta, \\ Salta, Argentina \\ ${ }^{2}$ National Institute of Chemical Engineering, INIQUI-CONICET, Universidad Nacional de Salta, \\ Salta, Argentina \\ Email: acostad@unsa.edu.ar
}

Received 28 February 2014; revised 25 March 2014; accepted 10 April 2014

Copyright (C) 2014 by authors and Scientific Research Publishing Inc.

This work is licensed under the Creative Commons Attribution International License (CC BY). http://creativecommons.org/licenses/by/4.0/

(c) (i) Open Access

\begin{abstract}
The current study presents the characterization of three types of materials after exposure to solar UV radiation. The selection of the materials is related to socio-economic aspects and rural activities in the northwest of Argentina (a.k.a. NOA). The objective was to establish a time parameter that allows estimating the durability of the materials according to their use. One of the materials is used as a protective cover (polyethylene film) of greenhouses, which are used for crop cultivation in the area. Another material is used in photovoltaic modules (PVMs), which are used to supply energy in isolated areas and the third material is sunscreen used by people that are exposed to UV radiation during rural activities. Degradation or deterioration of the materials was monitored using different spectroscopic assays: infrared was used to detect any structural changes in the material, X-rays were used for possible changes in the crystalline structure of the material, and spectral transmittance as this property is likely to be affected by changes in the structure of the material. The results showed that degradation of polyethylene seems to be related to the diminution in transparency and the loss of mechanical resistance with increasing exposure time. The decrease in transparency of one of the layers of a PVM caused a considerable decrease in the energy generated, whereas the sunscreens with a low sun protection factor (SPF) rapidly lost their protective properties and changed their photostability properties. Our results revealed a relationship between relative transmittance loss and exposure timse, which allows estimating the stability of different materials.
\end{abstract}

\section{Keywords}

Polyethylene Films, Photovoltaic Modules, Sunscreen Characterization, Degradation Effects, 


\section{Stability}

\section{Introduction}

The sun represents the main renewable energy source and only sends radiation energy to the earth, i.e. visible light, infrared and a lesser degree ultraviolet (UV) radiation. However, in the atmosphere, this radiation is converted into a variety of effects, some of which are important as energy resources, whereas others provoke material damage that has not been completely characterized yet and directly (body) or indirectly (resources) affect the population. Studies of this damage may help estimate the stability or durability of the materials and estimate their reposition or effect possible improvements. Therefore, three materials of different durability related to socio-economic aspects and rural activities in the northwest of Argentina (NOA) were selected. Ethylene vinyl acetate (EVA), a highly resistant material with a durability of more than 10 years, is used as sealant of photovoltaic panels. Another less resistant material (durability estimated in several years) is used to cover greenhouses and a third material with low resistance (durability in hours) which corresponds to sunscreens. The degradation or deterioration rate mainly depends on the UV radiation that affects the surface and the subsequent temperature of the material. Therefore, unexposed materials were compared with materials exposed to a high dose of UVC in a specially-designed chamber in order to establish a relationship between the damage provoked in the chamber and that provoked in the open air.

The Salta province is located in the northwest of Argentina; the Tropic of Capricorn cuts through provincial territory in the north. The extreme northern coordinate is $22^{\circ} 00^{\prime} \mathrm{S}$, and the southernmost coordinate is $26^{\circ} 23^{\prime} \mathrm{S}$. The easternmost coordinate is $62^{\circ} 21^{\prime} \mathrm{W}$ and the westernmost $68^{\circ} 33^{\prime} \mathrm{W}$. The average solar radiation in the Salta province, with the exception of the areas at higher altitude, is such that a material exposed for 10 years in the open air will have received an accumulated radiation on the working surface of approximately $3759 \mathrm{MJ} \mathrm{m}^{-2}$ and a UV radiation about $188 \mathrm{MJ} \cdot \mathrm{m}^{-2}$.

\subsection{Greenhouse Covering}

Greenhouses are a usual type of crop protection in the Salta region, which is characterized by frequent frost periods in winter. A typical example of a greenhouse is given in Figure 1. It is well known that when exposed to solar radiation several properties of these films suffer damage and after three years the films are totally degraded and they need to be replaced. Because of the price of this material per square meter and the large dimensions to be covered the cost of total reposition of the covering represents a great and significant economic effort. The covering assayed in the current study is a $150 \mu \mathrm{m}$ thick polyethylene film with anti-UV treatment commonly known as greenhouse plastic.

\subsection{Photovoltaic Modules}

Photovoltaic modules form a sandwich-type structure and consist of an upper protection layer, in this case a glass pane, and a highly transparent siliconized gel resistant to UV radiation. The modules assayed contained an upper layer of ethylene vinyl acetate (EVA), a photovoltaic cell, a siliconized gel and a final protective layer, a Tedlar $^{\circledR}$ polyvinyl fluoride (PVF) film. The current study presents several results obtained after deterioration of a photovoltaic panel after several years of sun exposure. The encapsulation material was analyzed to detect its deterioration. Figure 2 shows typical isolated rural equipment from the 1990s.

\subsection{Sunscreens}

Since years different products have been used to protect the skin from damage produced by the sun. External photoprotectors or sunscreens are pharmaceutical preparations that have the property to reflect, absorb or refract UV radiation from the sun or artificial sources to reduce the harmful action of sunrays. Zinc oxide $(\mathrm{ZnO})$ has been used for more than a century as an external skin protector. The first photoprotectors that were developed more than 40 years ago only contained UVB filters and were designed to prevent erythema. Currently, there exist formulae that contain inorganic compounds like titanium oxide, with combinations that provide broad-spectrum protection against UV and infrared radiation. However, their specific action varies and is under controversy. Physical characteristics of the target group (color and type of skin, hair, eyes, etc.) that help define 


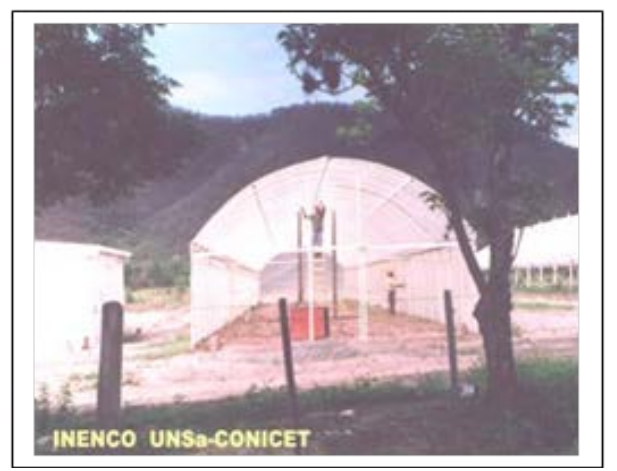

Figure 1. Typical greenhouse.

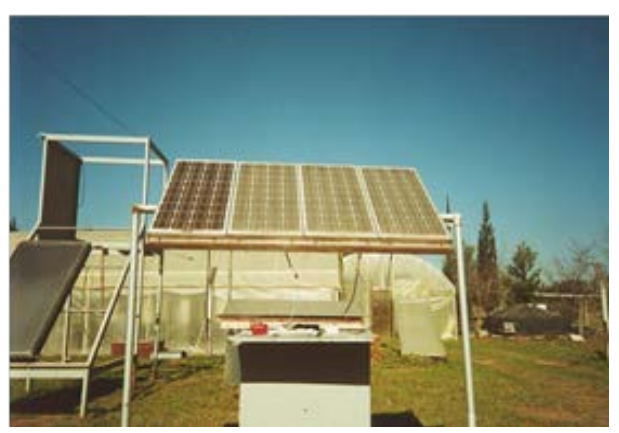

Figure 2. Four solar panels.

susceptibility to the sun and the tanning ability are also taken into account for the specific action of the blocker [1].

The concept of photostability is extremely important considering solar protection. Photostable substances define the SPF and protect the skin against prolonged exposure to the sun. Photolabile compounds weaken SPF, which may develop photoallergy. Photostability of the sunscreens can be studied by transmittance analysis followed by calculation of the ultraviolet protection factor (UPF) of both affected (UV-degraded) and unaffected samples. These samples may be either exposed in sun simulation chambers or directly to sunrays. A technique that yields complementary information is a parallel study of the same samples applying FTIR spectroscopy and both analyses allow the determination of the degree of instability of the material in order to estimate the reposition time.

\section{Experimental}

\subsection{Materials Assayed}

Greenhouse covering: Degradation of agro industrial polyethylene films, commercially produced by IPESA, was analyzed. This material can contain up to 5\% EVA, a copolymer of ethylene vinyl acetate. Besides EVA, the films contain certain minerals and other commercial anti-UV additives. The material is obtained by co-extrusion and contains three layers although the upper sides of the film do not differ much from one another. The thickness of the film is $150 \mu \mathrm{m}$.

Photovoltaic panels: The encapsulation material, which represents the contact between the cells and the glass of the silicon modules, is also EVA.

Sunscreens: Commercial sunscreens (Dermaglós ${ }^{\circledR}$ brand) with different SPF were assayed (Dermaglós ${ }^{\circledR} 8,15$, 30 and 60).

\subsection{Characterization Assays}

Transmittance assays $\left(T_{\lambda}\right)$ : The variation in transmittance between 650 and $700 \mathrm{~nm}$ is particularly important for the greenhouse covering, because photomorphogenesis effects occur within this spectrum [2]. The plastic sam- 
ple was analyzed by a HeNe laser that emitted at $638 \mathrm{~nm}$ and which was equipped with a $100 \mu \mathrm{A} \cdot \mathrm{W}^{-1}$ silicon detector cell. In the case of the photovoltaic collectors a series of transmittance curves were obtained after assaying the samples with a LI-COR 1800 spectrometer equipped with a global LI-COR 250 radiation detector. The spectral response of the sunscreens was directly measured with a specially designed UV transmittance analyzer with variable wavelength intervals $(1 \mathrm{~nm}, 5 \mathrm{~nm}$, etc.); the estimated value in each interval is the average for this interval.

UVA, UVB and visible light were measured using an optical spectrometer (OSM, Newport), operating within the 250 to $550 \mathrm{~nm}$ spectrum. The OSM is basically an OSM-100-UV spectrometer with a radiation source (LSM-DT-S), emitting deuterium (UV) and/or tungsten (visible $<550 \mathrm{~nm}$ ), an RS 232 interface, optical fibers and a cuvette holder. The sunscreen samples were irradiated with UV and the perpendicular radiation traversing the sample is detected. Transmittance spectra were obtained and further examined using OSM analyst software. An OSM cuvette holder was used as sample holder, which was isolated from any external radiation. The sample holder is a polished quartz cuvette, which is permeable to UV radiation. UPF determination through in vitro transmittance measures the transmission of the UV radiation through the sunscreen using an appropriate spectrometer. The assays were carried out according to the Australian/New Zealand standard (AS/NZS 4399:1996).

$X$-ray diffraction: Samples were analyzed using a powder X-ray diffraction equipment (Rigaku D/Max-IIC) with $\mathrm{Ni}$-filtered $\mathrm{Cu} \mathrm{K} \alpha$ radiation. Films of equal thickness were mounted on plates with identical irradiation areas with a scan rate of $2^{\circ} \theta \min ^{-1}$, a voltage of $40 \mathrm{kV}$ and a current of $20 \mathrm{~mA}$.

Fourier Transform Infrared (FTIR): IR spectra were carried out using FTIR Brücker equipment according to the multiple internal reflection (MIR) technique. This method allows detection of the presence of degradation products in analyzed samples. In the case of sunscreens the FTIR/HATR technique was used, which is Fourier transform infrared spectroscopy using a horizontal attenuated total reflectance accessory. The spectra were obtained with Perkin Elmer FTIR-Spectrum GX equipment which contains a horizontal $\mathrm{K}_{2} \mathrm{Se}$ cell. The sample holder consisted of a $2 \times 10 \mathrm{~cm}$ plate with a $4-\mu \mathrm{m}$-thick sunscreen layer and the spectrum was measured within a range of 4000 to $600 \mathrm{~cm}^{-1}$.

Degradation chamber: Because the durability or stability of the materials assayed highly differs it was necessary to simulate an accelerated degradation process, and therefore, materials were exposed in a degradation chamber under conditions that were adapted for each material. This was not necessary for the solar panels because the covering material was already degraded.

Simulation of the sun radiation within the spectrum of interest (UVR) was obtained by using an Osram Ultra-Vitalux lamp. The lamp was mounted on the upper part (ceiling) of a chamber, which isolated the lamp from the exterior and allowed control of the radiation intensity. Samples were placed at the base at a distance of 50 $\mathrm{cm}(20 ")$ from the lamp. The average UVB radiation intensity was $360 \mu \mathrm{W} \cdot \mathrm{cm}^{-2}$, which coincides with typical radiation values in the study area.

In order to avoid excessive heating of the chamber it was equipped with ventilation. The temperature measured at the spot where the samples were placed was about $40^{\circ} \mathrm{C}$. Sunscreen samples $\left(2 \mathrm{mg} \cdot \mathrm{cm}^{-2}\right)$ were uniformly distributed along a sample holder for analysis. The sample holder was a quartz plate of $1 \times 5 \mathrm{~cm}$. The transmittance spectrum was obtained with an OSM spectrometer equipped with an OSM cuvette holder.

\section{Results and Discussion}

\subsection{Greenhouse Coverings}

Spectral transmittance: Figure 3 shows the global spectral transmittance of a new polyethylene sample, a sample exposed during 3 years and another sample irradiated during 51 days in a TUV (tropospheric UV and visible radiation) degradation chamber as described above. It should be mentioned that the polyethylene sample irradiated for 51 days in the chamber was completely degraded with clearly visible cracks, and representative areas were selected for further assaying. It can be observed that transmittance in the UV region is higher for irradiated polyethylene than new, non-irradiated material, whereas in the visible and near-infrared region transmittance is quite similar. The reason for the difference in transmittance in the UV region is that degraded material has less capacity to absorb UV radiation as a result of the destruction of anti-UV additives. Although the global transmittance of the new and 3-year exposed protection covers did not vary significantly, the mechanical resistance to traction in degraded materials decreased considerably.

$X$-ray diffraction analysis: Figure 4 presents the results of a sample without exposure $\left(\mathrm{P}_{0}\right)$ and a sample from 


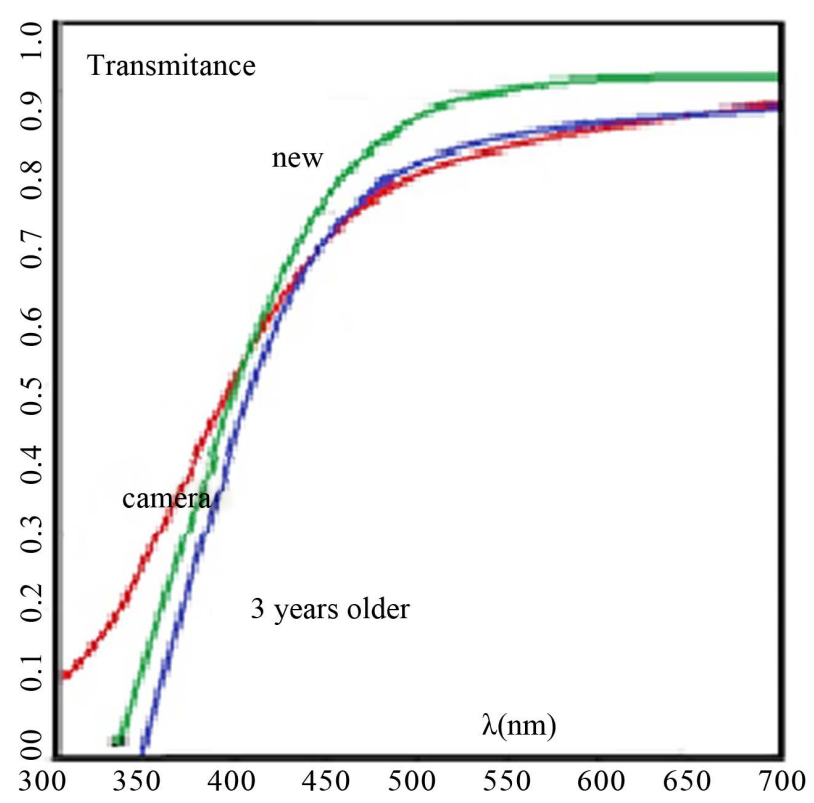

Figure 3. Global spectral transmittance of new polyethylene material (green line), material exposed for 3 years to the sun in the city of Salta (blue line) and irradiated material in a TUV chamber for 51 days (red line).

Samole Name: SO-INV-SAL

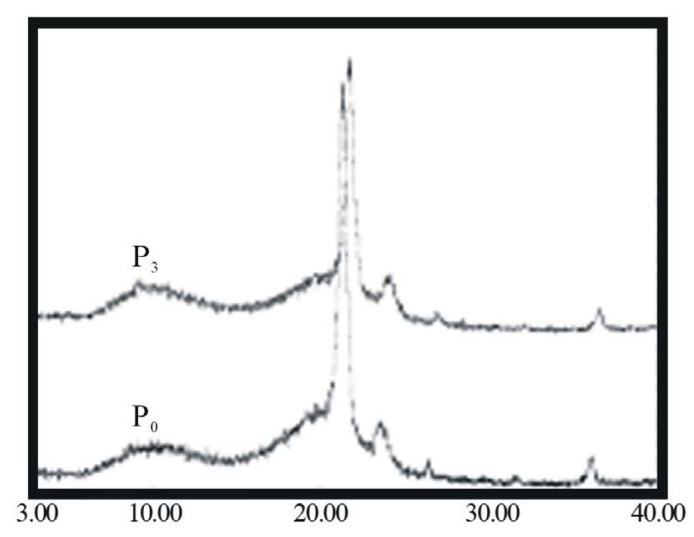

Figure 4. X-ray diffractograms of an unexposed sample $\left(\mathrm{P}_{0}\right)$ and of a sample from a greenhouse in Salta exposed during 3 years $\left(\mathrm{P}_{3}\right)$.

a greenhouse in the city of Salta exposed for 3 years $\left(\mathrm{P}_{3}\right)$. The crystalline peaks centered at $21.324^{\circ}$ and $23.560^{\circ}$

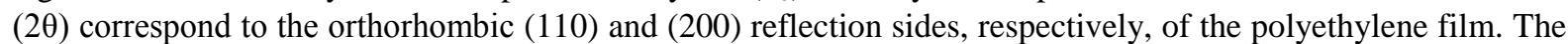
$2 \theta$ value increases when samples are exposed to radiation, which indicates a process that causes a decrease in the dimensions of the primitive or unit cell.

A lower value for $\mathbf{d}$ implies a higher degree of crystalline perfection. Several authors [3] have indicated that crystalline perfection is caused by the expulsion of chain parts that constitute ramifications of the principal chain of the polymer. Table 1 also shows the variation in the area of the peak with major intensity over the exposure time. An increase in the area compared with the background (which indicates the amorphous portion of the polymer) represents the degree of crystallinity of the sample, resulting in the percentage of crystallinity. The average height of the crystalline peaks provides information about the main dimension of the crystal which is perpendicular to the reflection side $\mathrm{D}$. $\mathrm{D}$ is related to the broadening of a peak according to the Scherrer equation: $\mathrm{D}$ $=\mathrm{k} \lambda / \beta \cos \theta$, where $\lambda$ is the $\mathrm{X}$-ray wavelength applied, $\theta$ the diffraction or scattering angle and $\beta$ corresponds to 
the line broadening at half the maximum intensity with a constant or shape factor, $k$, with a typical value of about 0.9 . From these results it may be inferred that the solar radiation process favors secondary crystallinity of coverings exposed to radiation.

FTIR (Fourier Transform Infrared) analysis: It is common to apply FTIR techniques to monitor degradation effects after exposure to UV radiation produced in diverse materials [3]. However, contrary to the methodology normally applied, i.e. obtain absorption or transmission spectra of the samples, the current study opted to use the MIR technique for two fundamental reasons. Firstly, the considerable width of the film gives very concentrated spectra, causing a loss of definition of the peaks. Secondly, UV radiation mainly causes superficial damage, and by using the reflection technique on the surface any alteration the material would have suffered will be maximized given the higher concentration.

Figure 5 shows the spectra within the 4000 to $400 \mathrm{~cm}^{-1}$ frequency interval, measured as absorption, of an unexposed sample (0) and samples exposed for 2, 5, 10 and 33 days. The absorption peaks of carbonyl groups (at approximately $1718 \mathrm{~cm}^{-1}$ ) become wider, which indicates the occurrence of degradation processes. This phenomenon is supported by the wide peaks, characteristic of -OH groups, and found in the $3300 \mathrm{~cm}^{-1}$ region. The presence of carbonyl groups in the covering is caused by the anti-UV additive, EVA. The size of the band at $1460 \mathrm{~cm}^{-1}$ change indicating a variation in the concentration of terminal methyl groups as a result of the rupture of the chains. The peaks at 720 and $730 \mathrm{~cm}^{-1}$ always appear when there are more than 4 consecutive methyl groups; the first peak $\left(720 \mathrm{~cm}^{-1}\right)$ is related to liquids and the $2^{\text {nd }}$ to solids. Monitoring of both peaks is very interesting because when they appear like in our case in a semi-crystalline polymer, one of them $\left(720 \mathrm{~cm}^{-1}\right)$ can be associated with the amorphous region and the other one with the crystalline region. The variation in relative height of the peaks provides information about the changes in the amorphous-crystalline aspect of the polymer and the degree of exposure of the material which supports observations with X-ray diffraction.

The relationship between the average heights of the double peak at $720 \mathrm{~cm}^{-1}$ with that of the peak at 1460 $\mathrm{cm}^{-1}$ is a way to quantify the amorphous-crystalline aspect [3]. An increase in the peak at $730 \mathrm{~cm}^{-1}$, the crystalline component, is observed with increasing exposure time, which confirms that one of the degradation effects is the rupture of the chains.

Damage to mechanical properties of the film: The polyethylene film used in greenhouses disintegrates after a few years of use because of degradation mainly caused by UV radiation of the sun. Traction assays of film irradiated in the chamber until its rupture revealed the following equation [4]:

$$
\mathrm{R}(\%)=99.9 \times \mathrm{e}^{-0.05 t c}
$$

This equation shows how the resistance of the material in the assay chamber diminishes over exposure time (tc expressed in days) [5]. This leads to the following relationship that explains the decrease in the film resistance:

$$
\text { In the city of Salta: } \mathrm{R}(\%)=99.9 \times \mathrm{e}^{-0.00116 t s}
$$

where ts corresponds to the open air exposure time (days) in Salta.

\subsection{Photovoltaic Panels}

Electrical characteristics: The unexposed and exposed modules were analyzed and current-voltage characteristics or I-V curves were made [1]. The results are given in Table 2 and Figure 6.

It can be observed that there exists a marked difference between both modules. The current of the open short circuit of the unexposed, new panel is $15.6 \%$ higher than that of the exposed panel. The voltage of the open circuit also varied although to a lesser extent as well as the parameters of the curve in general. Maximum power

Table 1. Crystalline parameters obtained after X-ray diffraction analysis of unexposed samples $\left(\mathrm{P}_{0}\right)$ and samples exposed for 3 years $\left(\mathrm{P}_{3}\right)$.

\begin{tabular}{cccccc}
\hline Sample & $2 \theta$ & $\mathrm{d}$ & Side & Crystallinity (\%) & $\mathrm{D}$ \\
\hline $\mathrm{P}_{0}$ & 21.320 & 4164 & 110 & 11 & 2.772 \\
$\mathrm{P}_{3}$ & 21.760 & 4.081 & 110 & 19 & 2.702 \\
$\mathrm{P}_{0}$ & 23.560 & 3.773 & 200 & & 2.370 \\
$\mathrm{P}_{3}$ & 24.120 & 3.687 & 200 & & 2.154 \\
\hline
\end{tabular}


Table 2. I-V results.

\begin{tabular}{cccccc}
\hline Panel & $\mathrm{V}_{\mathrm{OC}}(\mathrm{V})$ & $\mathrm{I}_{\mathrm{SC}}(\mathrm{A})$ & $\mathrm{V}_{\max }(\mathrm{V})$ & $\mathrm{I}_{\max }(\mathrm{A})$ & $\mathrm{P}_{\max }(\mathrm{W})$ \\
\hline Exposed $\left(\mathrm{P}_{1}\right)$ & 18.93 & 2.65 & 14.28 & 2.00 & 8.56 \\
Unexposed $\left(\mathrm{P}_{2}\right)$ & 19.40 & 3.14 & 14.60 & 2.80 & 40.88 \\
\hline
\end{tabular}

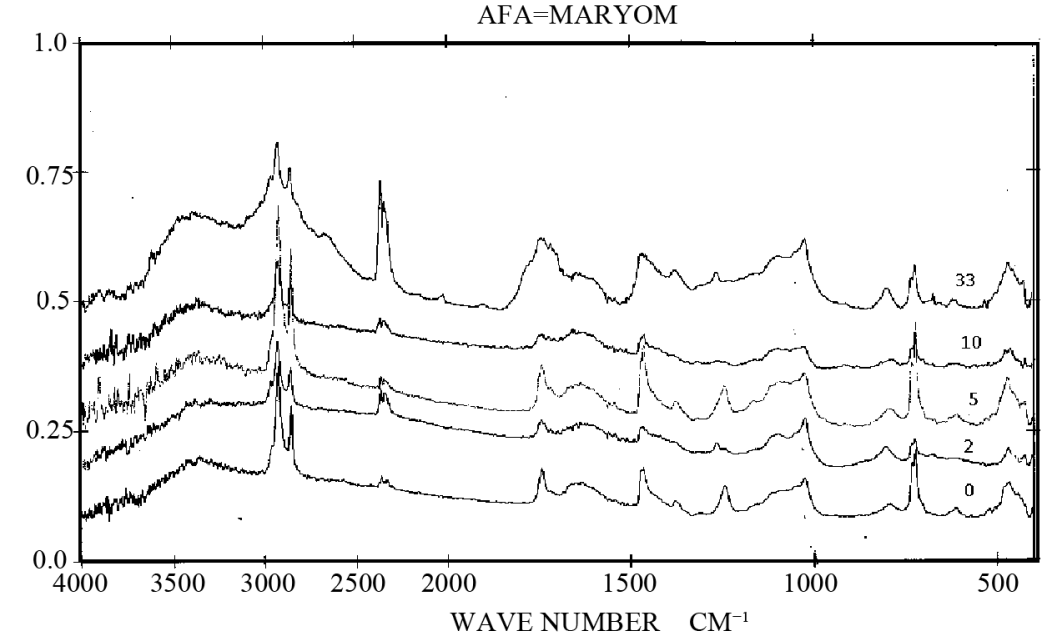

Figure 5. MIR absorption spectra.

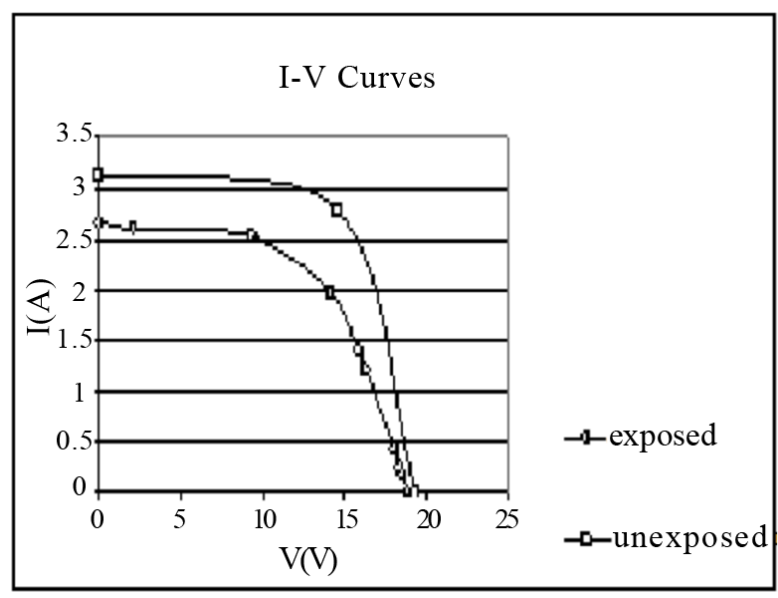

Figure 6. I-V curves of exposed and unexposed modules.

delivered by the exposed panel was $32 \%$ less than that of the unexposed panel.

Analysis of the transparent cover of the cells:

Optical characteristics: Spectral transmittance of EVA samples: Transmittance curves of EVA samples were constructed with the values obtained by spectrometry. Measurements were taken at different times of the day, but only two times are given as an illustration. The spectrometer was placed horizontally. Figure 7 shows that the transmittance is low at short wavelengths, increasing nearly linearly until $700 \mathrm{~nm}$ after which it becomes practically constant. It should be stressed that this spectral region the contribution of the radiation to the photovoltaic effect is much less than at longer wavelengths [6].

$X$-ray diffraction analysis: Diffractograms of the new and exposed materials were both amorphous, which allows concluding that whatever the effect of the radiation may be it would not be related to a morphological change or development of crystals in the material.

FTIR (Fourier Transform Infrared) analysis: Similar to the greenhouse coverings, the MIR technique was also applied in the case of the solar panels for the same reasons. Figure 8 demonstrates several changes that 
evidence the presence of degradation products. The band corresponding to the absorption of carbonyl groups becomes wider, indicating that similar to observations in the greenhouse coverings, other products like acid groups appear at lower frequencies (approximately at $1718 \mathrm{~cm}^{-1}$ ) after degradation. Appearance of the acid groups is confirmed by the wide band found at $3300 \mathrm{~cm}^{-1}$ and characteristic of -OH groups.

Intense peaks in the 1200 to $1020 \mathrm{~cm}^{-1}$ region corresponding to stretching of the ester group $\left(\mathrm{CO}-\mathrm{OCH}_{3}\right)$ are modified and lose resolution. This is in agreement with observations in the carbonyl region. The size of the band at $1460 \mathrm{~cm}^{-1}$ changes indicating a variation in concentration of the terminal methyl groups, probably due to the rupture of the chains.

Power loss: As can be shown in Figure 9 the results reveal that global transmittance of EVA did not vary with the variation in the zenithal angle. The global transmittance of the EVA material is practically constant at about $60 \%$, which coincides with spectral transmittance values measured with the spectrometer which are graphically presented in Figure 7. Figure 9 contains the results displayed in Figure 7 as well as the relative spectral response of a typical Si photovoltaic module in order to compare spectral transmittance of the EVA sample with the photovoltaic conversion curve [7].

According to these considerations, the Si solar cell assayed produced a response at wavelengths between 400 $\mathrm{nm}$ and $1100 \mathrm{~nm}$, with a maximum of around $850 \mathrm{~nm}$. Consequently, it can be deduced that the maximum current a panel can produce is obtained with radiation at a wavelength close to $850 \mathrm{~nm}$, which coincides with the wavelength spectrum characteristic of non-deteriorated EVA material. Figure 9 also shows that the highest loss of transmittance of deteriorated EVA is produced in the visible spectrum (350 to $650 \mathrm{~nm}$ ) where photovoltaic conversion is less. Therefore, the power loss is less than could be expected [8].

The performance of an operating solar cell is given by the ratio between the electrical energy produced and the solar energy intercepted by the surface. However, there exist certain factors that affect the performance of a solar cell to a higher or lesser degree. These factors may be internal or external like characteristics of the material, thickness of the wafer, active surface or geometry of the contacts. Environmental factors such as operational temperature and spectral composition of the incoming radiation may also be involved. This latter factor has been analyzed throughout the current study considering the deterioration of one of the components (EVA) of the module.

\subsection{Sunscreens}

Transmittance assay: The UPF is determined using a UVB and UVA transmission spectrophotometer. The CIE action spectrum for erythema (redness of the skin) is applied as a spectral weighting function for UV irradiation. Especially by including all wavelengths the real biologically effective radiance can be obtained. Figure 10 shows the incoming UV irradiation on a sunscreen sample and the incoming and transmitted erythemal spectra measured with an OSM-100-UV Newport spectrometer at the laboratory [9]. The UPF is defined as the ratio between the average transmitted erythemal UV radiation across air and average transmitted erythemal UV radiation across the sunscreen. Simultaneous transmittance assays and infrared spectrum measurements were carried out for sunscreens of the same brand but with different sun protection factors (SPF). In Figure 11 the transmitted radiation spectra for Dermaglós ${ }^{\circledR}$ sunscreen with different $\operatorname{SPF}(8,15,30$ and 65$)$ can be observed.

Table 3 shows the transmittance and erythemal transmittance (\%) in the UV, UVB and UVA range, protect-

EVA transmitance

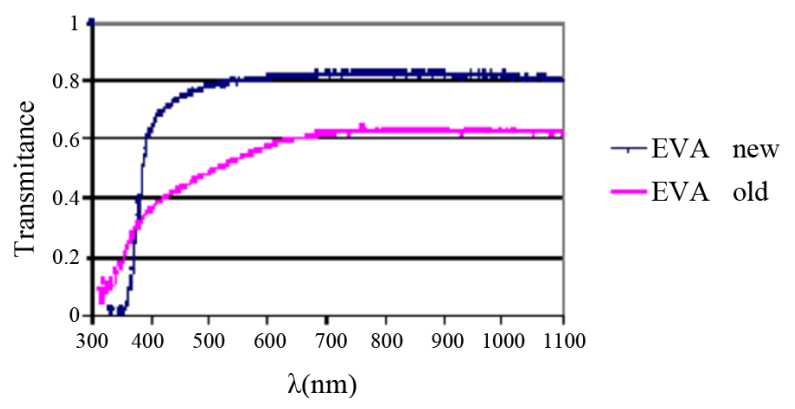

Figure 7. Transmittance of exposed (old) and unexposed (new) EVA samples. 


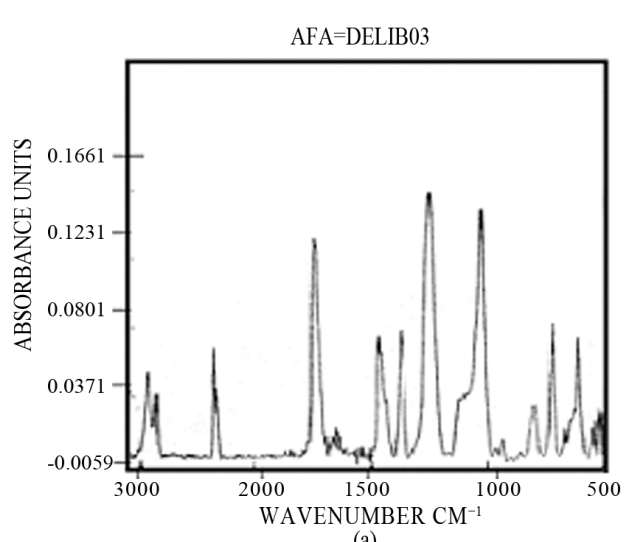

(a)

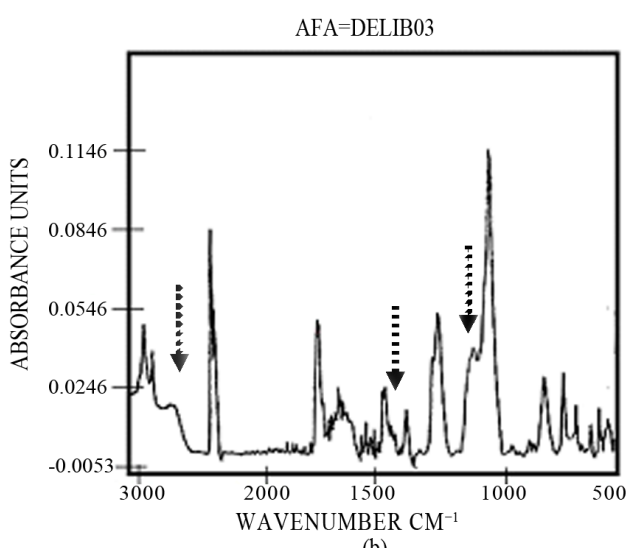

(b)

Figure 8. Infrared spectra (MIR) of the unexposed and exposed sample.

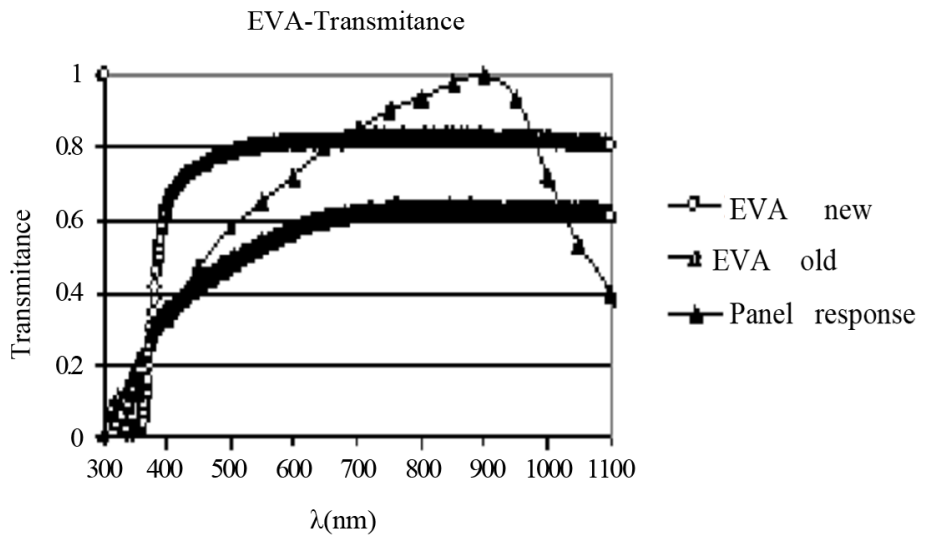

Figure 9. Comparative spectral transmittance.

Rad Incident.CIE.Eriemic incident

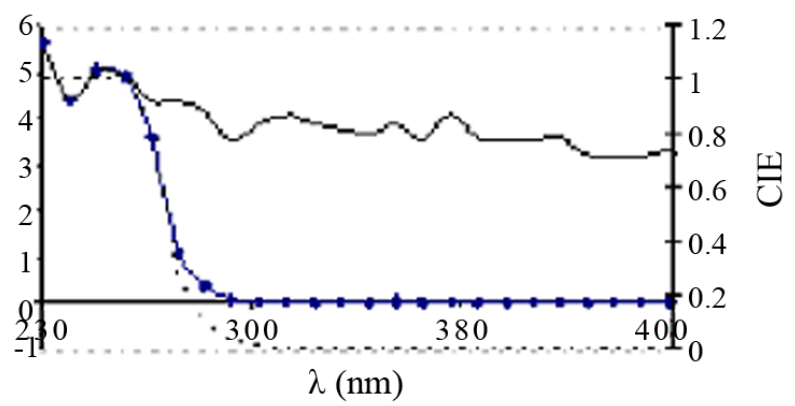

eriemic

incident $\cdots \cdots \cdot \mathrm{CIE}$

Figure 10. Normalized incoming irradiance, erythemal irradiance, IEC spectral irradiance and erythemal irradiance.

ion factors (UPF, UPF-B and UPF-A) calculated from the transmittance, and the transmittance (\%) in the visible spectrum until $550 \mathrm{~nm}$ (maximum wavelength of the spectrometer). Transmittance in the visible spectrum is a degree of the sunscreen protection [10].

The calculated UPF values agree with those mentioned on the label: For Dermaglós ${ }^{\circledR}$ sunscreen with SPF 8 (D8), the UPF was 8.25, D15 (UPF = 13.17), D30 (UPF = 33.29) and D65 (UPF = 66.34). Protection against premature photoaging caused by UVA is demonstrated by the percentage of transmitted radiation that varied 


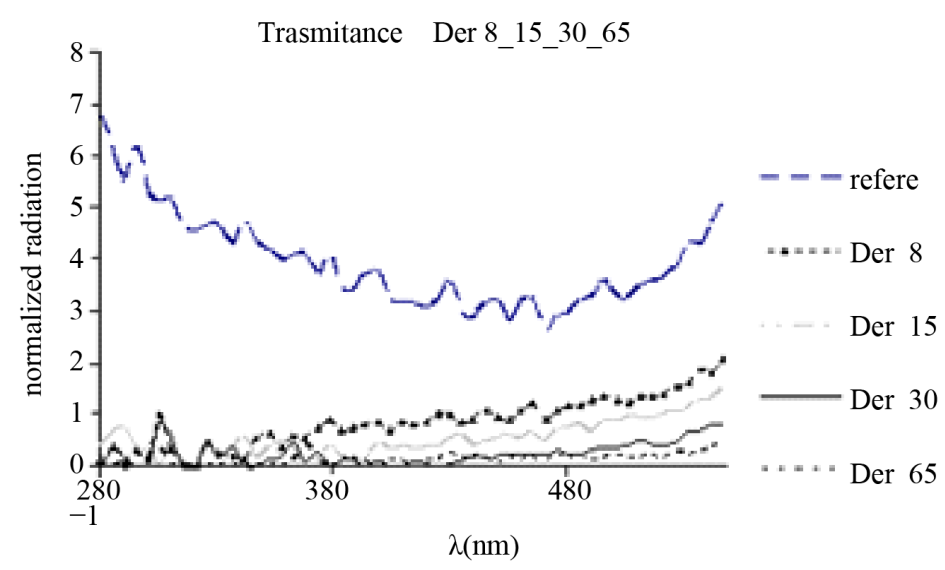

Figure 11. Incoming/transmitted radiation spectra for Dermaglós ${ }^{\circledR}$ sunscreen with different SPF.

Table 3. Transmittance (\%): Ratio of the incoming and transmitted radiation, ratio of global/erythemalradiation and UPF values for Dermaglós ${ }^{\circledR}$ sunscreens with different SPF (8, 15,30 and 65).

\begin{tabular}{ccccc}
\hline Radiation & D8 & D15 & D30 & D65 \\
\hline UV & 24.25 & 6.26 & 4.37 & 1.71 \\
UVA & 12.48 & 6.77 & 4.43 & 1.81 \\
UVB & 32.70 & 5.64 & 4.29 & 1.51 \\
ErythemalUV & 12.13 & 7.59 & 3.00 & 1.51 \\
ErythemalUVB & 12.30 & 7.59 & 3.00 & 1.51 \\
ErythemalUVA & 19.37 & 7.41 & 5.93 & 1.61 \\
Vis 400-550 & 48.11 & 21.57 & 9.28 & 8.48 \\
UPF & 8.25 & 13.17 & 33.29 & 66.34 \\
UPF-B & 8.26 & 13.17 & 33.34 & 66.35 \\
UPF-A & 5.16 & 13.49 & 16.87 & 62.24 \\
\hline
\end{tabular}

between 12.48 for D8 and 1.81 for D65 or by UPF-A, which is low in the case of D8 (5.16) and high in the case of D65 (62.24).

Photostability assays after degradation: An identical number of samples was simultaneously exposed to UV radiation in the degradation chamber [11]. Although the assay was carried out with all protection factors in order to exemplify the calculations and analyses only degradation of Dermaglós ${ }^{\circledR}$ SPF 8 and 65 will be shown below as they represent the extreme protection situations assayed. The total exposure time of the samples was 215 min. The sunscreens with low SPF, like D8, do not contain additives for protection against UVA. It can be observed that in no time transmittance of these creams in the UVA range increases from $12 \%$ to approximately $30 \%$. This confirms the fact that these sunscreens are classified as tanning lotions which means that they allow UVA to go through. Transmittance in the visible spectrum also increases from $27 \%$ to $48 \%$.

With the different UPFs calculated during the degradation and the corresponding exposure times a $2^{\text {nd }}$ degree polynomial correlation was observed with good correlation. This correlation, shown in Figure 12, is valid for the SPF range assayed and coincides with the manufacturer's recommendations regarding the period of effectiveness of each application [12].

Dermaglós ${ }^{\circledR} 65$ is labeled as Total Sunscreen and it is recommended for extremely sensitive skins with zero solar tolerance. Both sunscreens (D8 and D65) contain a physical protector (titanium oxide) in their formula, assuming a higher photostability than the indoor tanning lotions that do not contain this compound. The transmittance analysis of sun exposure times close to $500 \mathrm{~min}$ and a posterior calculation of the UPF revealed a polynomial regression with a good correlation coefficient (Figure 13). It can be seen that Dermaglós ${ }^{\circledR} 65$ was very stable until 300 min; after that time the UPF decreased but it still maintains a UPF within the range of excellent sunscreens. 


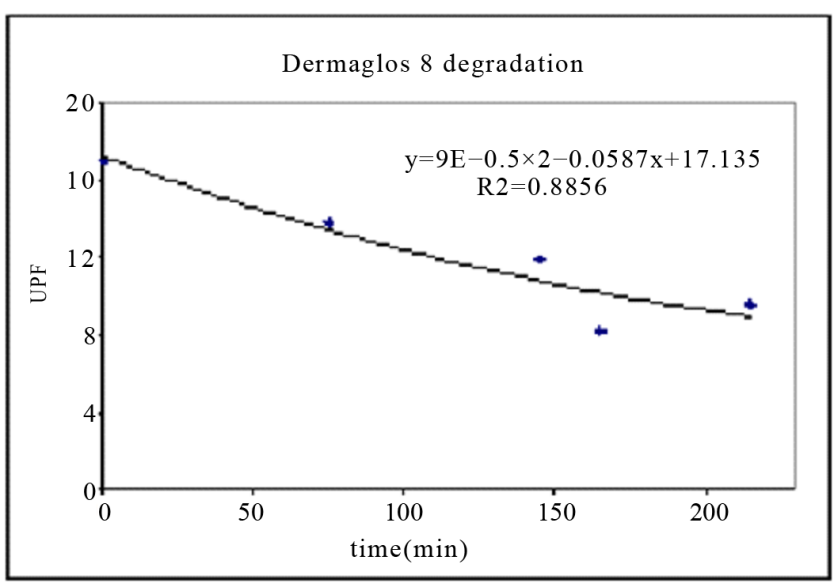

Figure 12. Polynomial regression of degradation of Dermaglós ${ }^{\circledR} 8$.

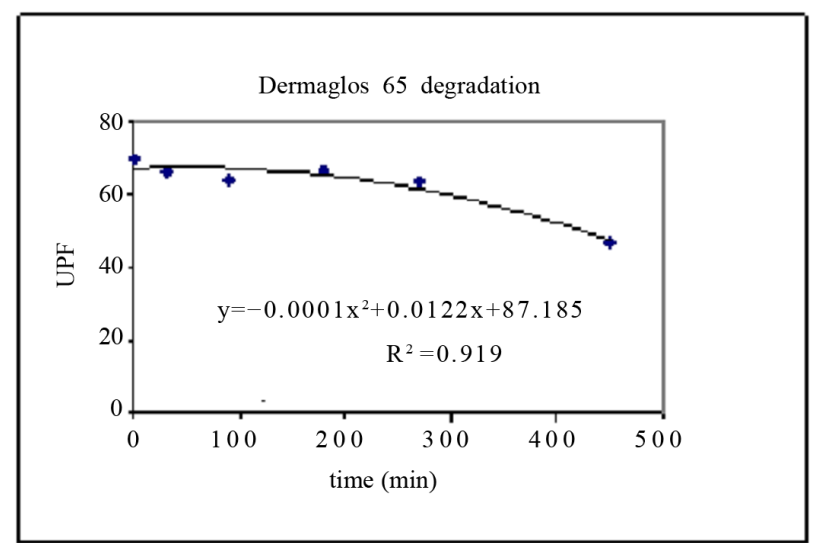

Figure 13. Polynomial regression of degradation of Dermaglós ${ }^{\circledR} 65$.

FTIR assays: Infrared spectroscopy assays were carried out with the whole series of sunscreens. Samples were exposed for different times to degradation by direct solar radiation and in the UV chamber. For samples with a high protection factor, like SPF 65, the serial spectra of different exposure times to direct solar radiation and in the UV chamber did not show great variations after short exposure times. Only after eight hours changes, which will be discussed below, could be observed (Figure 14). Modifications were produced in a peak at 1620 $\mathrm{cm}^{-1}$, which appeared as a new signal (a shoulder) in the product after exposure to sunlight. This alteration is associated with a change in conjugated ester bonds present both in octocrylene and Parsol MCX and they traditionally constitute the sites sensitive to photochemical modifications. Another sign of a modification after sun exposure is displayed in the peak at $1284 \mathrm{~cm}^{-1}$, which disappears in the exposed spectrum. Finally, the peak at $600 \mathrm{~cm}^{-1}$, observed in sunscreens with higher protection, proves existence of $\mathrm{TiO}_{2}$ because of an elevated baseline with a wide curvature. $\mathrm{TiO}_{2}$ was also confirmed by an independent assay in which the sunscreen was burned at $700^{\circ} \mathrm{C}$ to destroy the organic compounds.

In the case of SPF 8 sunscreen after exposure to solar radiation under exactly the same conditions as the maximum protection studied (SPF 65) changes were notorious. A prominent diminution is observed in the band in the region of vibrations of the -OH groups $\left(3500 \mathrm{~cm}^{-1}\right)$ after exposure to solar radiation, attributable to an evident dehydration process. The modifications are similar to those observed in SPF 65, but the most important changes are those observed in the $800 \mathrm{~cm}^{-1}$ region. In this region, dominated as the fingerprint of the spectrum, a change in the profile is proof of a change in the structure of the material, interpreted as evidence of a degradation process.

$\mathrm{TiO}_{2}$ absorption is superimposed with degradation products in the fingerprint region, but because the SPF 8 formula does not contain $\mathrm{TiO}_{2}$ it does not hide any changes, as is the case in SPF 65. The modifications men- 
tioned can be observed in Figure 15.

The results of the three materials after exposure to solar UV radiation (either in the chamber or in the open air) allow the construction of a curve of the loss of transmittance (\%) as a function of the exposure time (Figure 16). Adjustments by regression make it possible to estimate the durability of the materials, determining a reapplication frequency in min for sunscreens, and replacement after three years for the greenhouse coverings and 10 years for photovoltaic modules.

\section{Conclusions}

\subsection{Protection Layers}

Polymers with a structure similar to Agrotileno ${ }^{\circledR}$ (LLDPE type) underwent degradation effects and structural changes when they were exposed to UV radiation, either artificially in a UV chamber or in the open air (greenhouses). The process mainly leads to an increase in the crystalline proportion of the material, as can be inferred from X-ray diffraction assays. This increase is associated with a photo-oxidative rupture process that degrades polymer chains that are afterwards recombined, as can be inferred from FTIR. The most notable consequence is manifested in the decrease in transmittance which is related to a structural change that modifies the physical, mechanical and optical properties of the material. Transmittance in the UV range is higher for irradiated polyethylene than for new material, but at wavelengths above $425 \mathrm{~nm}$ transmittance is practically the same in both cases. This can be attributed to the fact that the degraded material has less capacity to absorb UV radiation due to the destruction of anti-UV additives. Although the global transmittance of new and 3-year-exposed coverings

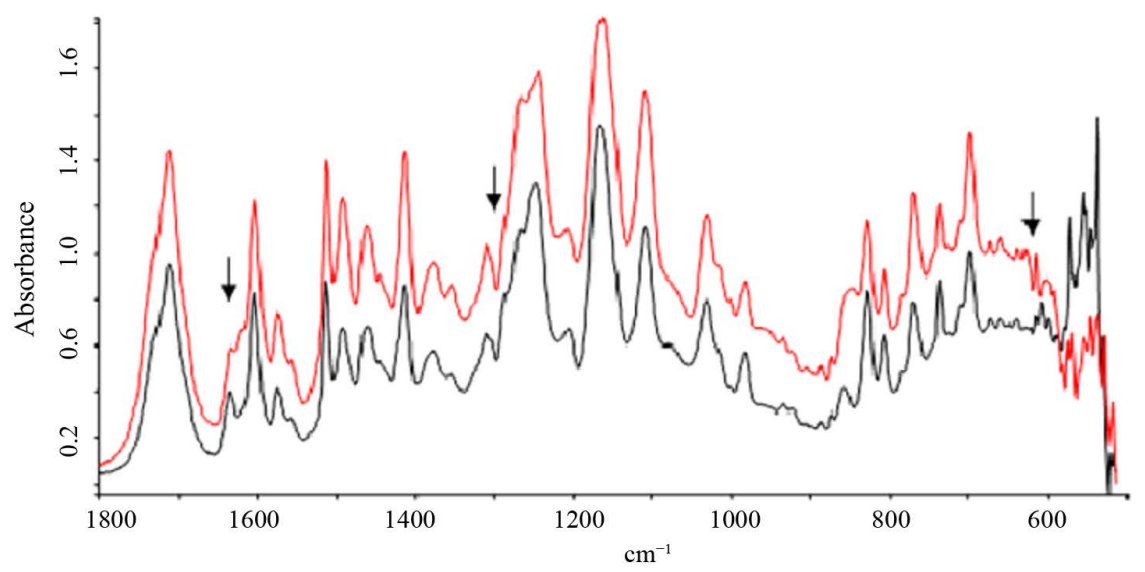

Figure 14. FTIR/HATR spectra of SPF 65 sunscreen without exposure (black line) and after $8 \mathrm{~h}$ of exposure (red line).

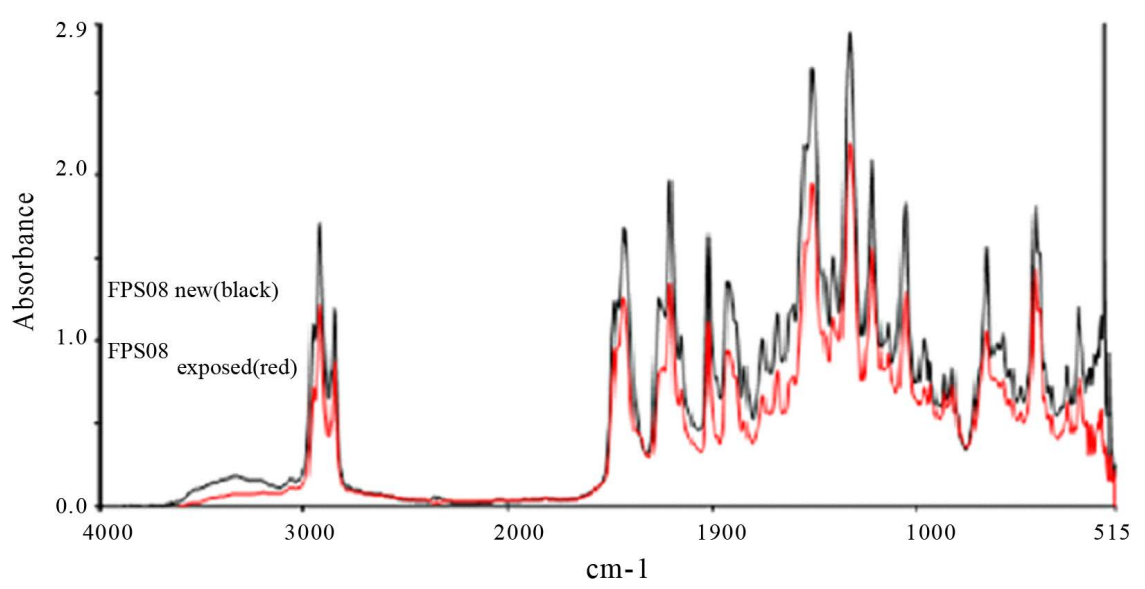

Figure 15. FTIR/HATR spectra of SPF 8 sunscreen without exposure to the sun (black line) and exposed to the sun (red line). 


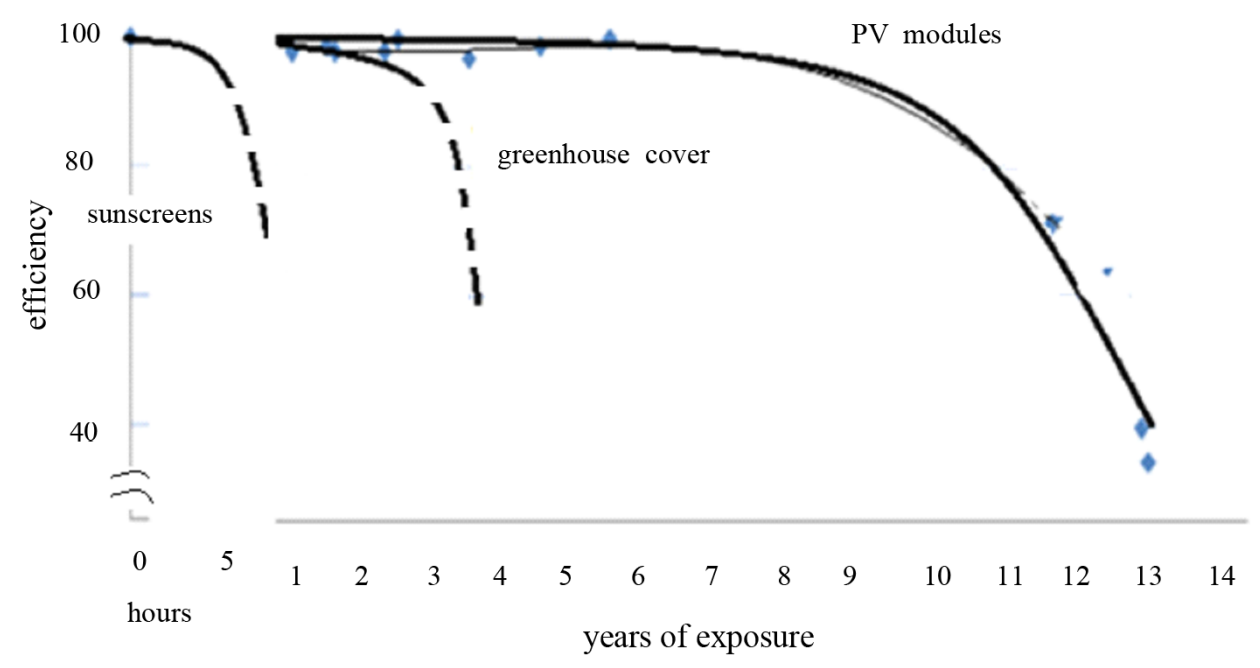

Figure 16. Transmittance loss curves.

did not vary significantly, the mechanical resistance (traction) in the degraded material diminished considerably. It is important to stress that the loss in transmittance capacity of the material is less than $12 \%$, even after 51 days of exposure in the chamber, and less than $16 \%$ after exposure in the open air for three years. Even though this loss $(16 \%)$ is not significant for agricultural applications, from a mechanical point of view however, it is inacceptable.

\subsection{Photovoltaic Panels}

Prolonged exposure of these devices in the open air caused degradation effects with regard to the properties of the materials it is composed of. This process was manifested in a progressive power loss. The main cause of the power loss of the panels is the degradation of the protection layer which is generally composed of silicons (in this particular case EVA). This causes a decrease of about $20 \%$ in its transmittance in the near-infrared and visible range, varying about $40 \%$ in wavelengths around $400 \mathrm{~nm}$ and around $20 \%$ in wavelengths near $700 \mathrm{~nm}$. This decrease in transmittance consequently leads to a diminution in the generated current (performance), because it is directly related to the radiation that reaches the electricity-producing cell. The power loss of the modules caused by this effect is about 30\%. The infrared assay (FTIR) showed degradation products that originate from the rupture of the polymer chains, which produce acids that may contribute to the oxidation of the contacts. $\mathrm{X}$-Ray diffraction analysis demonstrated that the material is still amorphous even after degradation.

\subsection{Sunscreens}

The photoprotective characterization of the different sunscreen formulae can be determined by UV transmittance and FTIR assays. The FTIR/HATR technique represents an efficient and precise study to rapidly determine presence of active components mentioned in the formulae. This technique also results adequate to monitor the photostability of the material when exposed to radiation, directly or simulated in the UV chamber. The degradation curves obtained by polynomial regression confirm more photostability in sunscreens with higher SPF, while sunscreens with lower SPF are less stable. Sunscreens with a SPF factor of 30 and 65 were classified as highly stable while those with factors 8 and 15 were classified as little stable. Sunscreens with little stability should be reapplied before the two hours indicated by the manufacturer.

In synthesis, the polymers used in the greenhouse coverings and the photovoltaic panels present effects associated with degradation types like radical or homolytic fission in the constitutive chains, while sunscreens with a SPF of 30 and over use inorganic ingredients that are more resistant against UV degradation. The assays with the three materials were carried out in Salta, Argentina, and allow the following statements with regard to durability:

1) The loss of transmittance of the greenhouse coverings did not represent a determining factor in the durability but in the mechanical deterioration. UV treatment in a simulation chamber allowed assessing to a high degree of certainty the performance of the same material in the open air. The average lifetime after 51 days in the 
chamber equaled three years in the open air in the city of Salta.

2) The power loss in the photovoltaic modules associated with damage to the EVA polymer played a major role, and the findings of the current study demonstrate that this factor is very important after exposure in the open air for more than 10 years. The current study did not measure the damage to electrical parameters produced by UV exposure.

3) Finally, sunscreen with a high SPF (like Dermaglós ${ }^{\circledR}$ 65) conserved its protection capacity until 300 min after its application, which agrees with the recommendations for these high FPS sunscreens for people exposed to solar radiation.

\section{References}

[1] MacKie, R.M. (2000) Effects of Ultraviolet Radiation on Human Health. Radiation Protection Dosimetry, 91, 15-18. http://dx.doi.org/10.1093/oxfordjournals.rpd.a033186

[2] Andrady, A.L., Pegram, J.E. and Tropsha, Y. (1993) Changes in Carbonyl Index and Average Molecular Weight on Embrittlement of Enhanced Photodegradable Polyethylene. Journal of Environmental Degradation of Polymers, 1, 171-179.

[3] Guadagno, L., et al. (2001) Chemical and Morphologial Modifications of Irradiated Linear Low Density Polyethylene (LLDPE). Polymer Degradation and Stability, 72, 175-186. http://dx.doi.org/10.1016/S0141-3910(01)00024-6

[4] Tilca, F.D., Cadena, C. and Vicente, M. (2001) Ensayos de films de polietileno de baja densidad para invernaderos expuestos a altas dosis de radiación UV. Revista de la ASADES (Asociación Argentina de Energía Solar), Avances en Energías Renovables y Medio Ambiente-AVERMA, 4, 8, 127-131.

[5] Tilca, F. (2002) Diseño y construcción de una cámara de ensayos de radiación ultravioleta para estudiar la degradación de materiales poliméricos. Tesis de Maestría en Energías Renovables, Facultad de Ciencias Exactas, UNSa.

[6] Duffie, J.A. and Beckman, W.A. (2006) Solar Engineering of Thermal Processes. 3rd Edition, Wiley Interscience, New York, 747-775.

[7] Montero, M. (2003) Evaluación y análisis del comportamiento eléctrico de paneles fotovoltaicos expuestos a la intemperie por más de 10 años. Tesis de Maestría en Energías Renovables, Facultad de Ciencias Exactas, UNSa.

[8] Pern, F. and Glick, S. (2000) Photo Thermal Stability of Encapsulated Si Solar Cells and Encapsulation Materials upon Accelerated Exposures. Elsevier.

[9] McKinlay, A.F. and Diffey, B.L. (1987) A Reference Action Spectrum for Ultraviolet Induced Erythema in Human Skin. CIE Journal, 6, 17.

[10] González, S., Fernández-Lorente, M. and Gilaberte-Calzada, Y. (2008). The Latest on Skin Photoprotection. Clinics in Dermatology, 26, 614-626. http://dx.doi.org/10.1016/j.clindermatol.2007.09.010

[11] Serpone, N., Dondi, D. and Albini, A. (2007). Inorganic and Organic UV Filters: Their Role and Efficacy in Sunscreens and Suncare Products. Inorganica Chimica Acta, 360, 794-802. http://dx.doi.org/10.1016/j.ica.2005.12.057

[12] Suarez, H., Acosta, D. and Cadena, C. (2012) Analisis de la capacidad de fotoproteccion de una crema protectora solar aplicada a formulaciones no comerciales. Energias Renovables y Medioambiente, 30, 63-70. 